Equinoccio. Revista de psicoterapia psicoanalítica, 2(2), julio-diciembre 2021, pp. 123-133.

ISSN: 2730-4833 (papel), 2730-4957 (en línea). DOI: doi.org/10.53693/ERPPA/2.2.7.

\title{
ABSTINENCIA Y NEUTRALIDAD \\ DE JORGE ROSA
}

\author{
Adenda por \\ Rosario Allegue \\ Asociación Uruguaya de Psicoterapia Psicoanalítica \\ Montevideo, Uruguay \\ Correo electrónico: rosarioallegue@gmail.com \\ ORCID: 0000-0003-1584-425X
}




\section{Sobre el texto y su autor}

El artículo que incluimos en esta sección de relecturas, escrito por el Dr. Jorge Rosa (1948-2009), se publicó originalmente en el primer número del tercer tomo de la Revista de Psicoterapia Psicoanalítica, de setiembre de 1989. Al publicar un artículo suyo, que refiere al eje temático de esta entrega de Equinoccio, recordamos también, en su persona, al núcleo fundador de AUDEPP.

Jorge Rosa fue doctor en Medicina con posgrado en Psiquiatría. También había realizado estudios de psicología. Desde su etapa de estudiante, optó por una perspectiva psicoanalítica de la subjetividad.

En 1981 fue parte de la creación de AUDEPP; asumió, de hecho, la primera presidencia. La institución se propuso desde el comienzo como un lugar de debate y de intercambio, de libertad y de respeto por las preferencias teóricas de cada uno de sus miembros. A él le tocó encabezar ese período inicial, en el que se fue plasmando una identidad profesional y una concepción de la salud mental, ambas con fuerte impronta social.

Fue también animador decidido en la fundación de la Federación Latinoamericana de Asociaciones de Psicoterapia Psicoanalítica y Psicoanálisis (FLAPPSIP), que tuvo lugar en Montevideo en 1998. En esa ocasión, también fue elegido como su primer presidente.

Con la creación del Instituto Universitario de Posgrados de AUDEPP (en su etapa como IPPA), fue integrante del primer Consejo Académico y docente a cargo del Módulo de Freud. El instituto lleva actualmente su nombre.

Sus intereses variados y sus amplísimas lecturas lo llevaron a transitar por la historia, la ciencia, la política y la cultura. Desde 2004 hasta su muerte, coordinó un ciclo de cine en AUDEPP, que muchos recuerdan como el descubrimiento de una nueva forma de entender el arte y los procesos humanos.

Su último trabajo, presentado en 2009 en el V Congreso de FLAPPSIP, que también presidió, se refiere a la teoría de la evolución en su articulación con la obra de Freud y sintetiza mucho de su pensamiento, interdisciplinario y dialéctico. 


\section{INTRODUCCIÓN}

Desde que el psicoanálisis es tal, es decir, desde que se abandonó la sugestión, la regla de abstinencia enunciada por Freud ha sido uno de los elementos básicos de la técnica. Junto a esta regla de abstinencia se menciona siempre el concepto de neutralidad, que no fue denominado de este modo por Freud (1988e), sino que se va desarrollando en referencia a la cura, como una actitud que caracteriza al analista durante la misma.

Un primer planteo es si estos conceptos, manejados habitualmente como sinónimos, lo son realmente o si son sustancialmente distintos.

Schkolnik (1987) plantea que la especificidad del vínculo pacienteanalista está dada por la regla de abstinencia, caracterizándola desde el punto de vista descriptivo por dos condiciones:

- Una limitación de la neutralidad del analista.

- El mantenimiento de la neutralidad del analista.

Para esta autora, la regla de abstinencia no se cumple nunca en forma estricta, de tal forma que el análisis bascula entre la transgresión y la abstinencia.

Baranger y Baranger (1969) señalan que el analista en su actuación terapéutica debe someterse a lo que se podría llamar «la regla de abstención ideológica», que diferencia de la actuación pedagógica y que podría definirse de esta manera: «El psicoanalista debe abstenerse de toda influencia sobre el analizando en el campo ideológico», regla que para él es estrictamente inaplicable. Desde nuestro punto de vista, abstinencia y neutralidad son conceptos distintos. 
La abstinencia es un elemento de tipo técnico, condición sine qua non para el desarrollo del proceso analítico. La neutralidad, en tanto implica una ausencia ideológica, es insostenible. Intentaremos precisar estos conceptos.

\section{ABSTINENCIA}

Comenzaremos tratando de delimitar qué se entiende por abstinencia en la concepción freudiana. En Puntualizaciones sobre el amor de transferencia (Freud, 1988c) leemos que:

La cura tiene que ser realizada en la abstinencia [...]. Yo quiero postular este principio: hay que dejar subsistir en el enfermo necesidad y añoranza como unas fuerzas pulsionantes del trabajo y la alteración, y guardarse de apaciguarlas mediante subrogados. (s.p.)

En Nuevos caminos de psicoterapia psicoanalitica Freud (1988d) enuncia el principio soberano en el campo de la técnica:

En la medida de lo posible, la cura analítica debe ejecutarse en un estado de privación, de abstinencia. Por abstinencia no debe entenderse la privación de una necesidad cualquiera —eso sería desde luego irrealizable — ni tampoco lo que se entiende por ella en el sentido popular, a saber, la abstención del comercio sexual; se trata de algo diverso, que se relaciona más con la dinámica de la contracción de la enfermedad y el restablecimiento. (s.p.) 
Así planteada, como motor del trabajo analítico, «la privación impide que la cura se convierta en satisfacción sustitutiva, permitiendo así que se desarrolle el proceso del análisis» (Freud, 1988d, s.p.).

\section{NEUTRALIDAD}

Laplanche y Pontalis (1971) definen la neutralidad como una de las cualidades que caracterizan al analista durante la cura, señalando que el mismo debe ser neutral en cuanto a los valores religiosos, morales y sociales; también en cuanto a las manifestaciones transferenciales, así como al discurso del paciente, no jerarquizando a priori determinado fragmento o determinado tipo de significaciones.

El concepto de neutralidad surge de los trabajos de Freud sobre la técnica psicoanalítica. En Consejos al médico sobre el tratamiento psicoanalítico (1988a) aparece la imagen del analista como un cirujano que debe llevar a cabo su función tan hábilmente como le sea posible, es decir, alguien que operaría en un campo determinado, prescindiendo de lo que está fuera de él. Asimismo, señala que la ambición pedagógica es tan inadecuada como la ambición terapéutica.

En La iniciación del tratamiento, Freud (1988b) relaciona el establecimiento de una transferencia operativa, un rapport en regla, con el concepto de neutralidad analítica. La primera meta es allegar al paciente al mismo y, por ende, a la persona del médico. Para ello es necesario darle tiempo. Se puede hacer fracasar esta meta si se adopta una actitud diferente a la empática, por ejemplo, una actitud moralizante, o si se comporta como el representante o mandatario de un tercero.

En Los caminos de la psicoterapia psicoanalítica, Freud (1988d) rechaza la idea de considerar al paciente como un bien propio y, por lo tanto, 
formar su destino, inculcarle las ideas del analista o modelarlo a la imagen del mismo con el orgullo de un creador.

\section{DISCUSIÓN}

La abstinencia es un concepto técnico. Es un elemento del encuadre analítico. No comunicamos al paciente cosas personales, nuestra visión de los hechos, nuestras ideas. No hacemos, no actuamos con el paciente de acuerdo a los deseos de este o a los nuestros planteados desde la contratransferencia. Si accediéramos a los pedidos de amistad, de amor, de contacto extraclínico se produciría una alteración en la dinámica transferencial con dificultad para el conocimiento de lo reprimido inconsciente.

Pero ¿qué es la neutralidad? Pregunta Castel (1980): «¿Mediante qué procedimientos un personaje nuevo, el psicoanalista, se instala en el privilegio ambiguo de su neutralidad?» (s.p.).

Castel (1980) aborda desde distintos ángulos su hipótesis básica: el poner de manifiesto las consecuencias que implica el carácter contractual del psicoanálisis, y uno de estos ángulos es la convención de la neutralidad. Si todo el psicoanálisis se inscribe dentro de un contrato, esta estructura no es un marco con un contenido dentro, sino la matriz productora de los efectos psicoanalíticos.

La situación analítica aparece como una convención (arbitraria y necesaria) justificada para acceder al inconsciente (o al conocimiento teórico de alguno de sus efectos). Esta convención exige poner entre paréntesis los determinismos políticos y sociales y dejar de lado la realidad. La relación entre lo analítico y lo extraanalítico se da de antemano, la convención de la neutralidad opera tratando de neutralizar lo que en la realidad nunca es neutro. 
Y nosotros nos preguntamos: ¿mediante qué procedimientos este personaje, el psicoanalista, puede definirse como carente de ideología?, ¿cómo conceptualiza el psicoanálisis?, ¿cómo vive su identidad?

La neutralidad implica un vacío ideológico. Nos referimos a ideología al decir de Grinberg y Grinberg (1976), como «una manera de ver al mundo en función de una convicción sociopolítica valorativa de los vínculos existentes entre los individuos y la sociedad a la que pertenecen. Estas convicciones incluyen en cada individuo fantasías inconscientes específicas» (s.p.).

Dicen Baranger y Baranger (1969) que el psicoanálisis, como toda ciencia, es una ideología, y Grinberg y Grinberg (1976) sobre este punto hablan de ciencia ideologizada. Lo que es claro es que no podemos pensar en la posibilidad de ejercer una práctica que nos vincula con el otro como ser específico, que esté desprendida de toda nuestra concepción en torno a los objetos comunes en los cuales estamos insertos ese otro y nosotros, y los elementos vinculares que esto implica.

Las fantasías inconscientes, que también son parte de nuestra identidad, estarán presentes desde el momento mismo en que atendemos una llamada telefónica, abrimos la puerta, tendemos la mano, y luego durante todo el trabajo analítico. Todo está relacionado con qué vivencia tengamos de nuestra identidad profesional, parte integrante de nuestra identidad. Como analistas tenemos una ideología científica, es decir, concebimos nuestra tarea inscripta en un sistema de valores o representaciones más o menos conscientes.

Achard (1972) sostienen que aislarse y prescindir del proceso histórico-social, lejos de constituir una actitud neutral, es un modo activo de tomar posición. Para ellos hay dos posibilidades teórico-técnicas a discutir. Por un lado, no considerar la realidad externa y, cuando el paciente la trae, interpretar el nivel transferencial y los objetos internos. La otra actitud posible es incluir el fenómeno en el campo y tratarlo, además de los campos mencionados, como un objeto común al paciente y al analista. 
Langer (1984) señala que la realidad social se filtra a través del discurso del paciente y, lo quiera o no el analista, a través de sus interpretaciones. Por lo tanto, mantener el concepto de neutralidad como válido y posible, es en sí manifestación de una ideología conservadora.

Lo antedicho nos lleva de la mano a reflexionar sobre los criterios de curación. Baranger y Baranger (1969) señalan que la curación es una actitud normativa. Seguramente cada uno de nosotros en nuestra práctica considera que la curación existe o no, pasa por determinados cambios $u$ otros. Y esto va a estar determinado por la concepción de salud, enfermedad, adaptación o desadaptación, libertad individual y colectiva, sexualidad, presente en cada uno de nosotros, que son elementos cargados de ideología. Todo esto parece muy claro si lo referimos a la concepción de normalidad. Incluso ante el síntoma, a la patología «indiscutible», le vamos a dar un lugar distinto según conceptualicemos qué lugar ocupa este en el devenir histórico y social del individuo concreto.

Por lo tanto, desde nuestro punto de vista no existe la interpretación ingenua. Para Baranger y Baranger (1969), la relación que implica una interpretación es de por sí una relación ideológica.

Concluimos, por lo tanto, que abstinencia y neutralidad son conceptos distintos. La abstinencia es una posibilidad técnica, una cierta posibilidad. La neutralidad es una ficción. Es una ficción creer que en nuestra relación con el otro podemos prescindir de nuestros sistemas valorativos y relacionales. El peligro de esta ficción es que su desconocimiento, su escotomización nos hace ser menos «neutrales».

Podríamos decir, entonces, que el conocimiento de nuestra no neutralidad nos hace ser más conscientes de que en el señalamiento estamos optando también, aun a nuestro pesar, por una determinada concepción de nuestros objetivos, y esto está inextricablemente ligado a las ideas predominantes en un determinado tiempo histórico, de lo que ningún ser humano logra excluirse. 


\section{REFERENCIAS BIBLIOGRÁFICAS*}

AchARD, L. (1972). Crisis social y situación analítica. Cuestionamos 1. Granica.

Baranger, W. y Baranger, M. (1969). Interpretación e ideología: sobre la regla de abstención ideológica. En Problemas del campo psicoanalítico. Kargieman.

Castel, R. (1980). El psicoanalismo, el orden psicoanalítico y el poder. Siglo XXI.

Freud, S. (1988a). Consejos al médico en el tratamiento psicoanalítico. En Obras completas (vol. XII). Amorrortu.

FREUd, S. (1988b). La iniciación del tratamiento. En Obras completas (vol. XII). Amorrortu.

FREUd, S. (1988c). Puntualizaciones sobre el amor de transferencia. En Obras completas (vol. XII). Amorrortu.

FREUd, S. (1988d). Los caminos de la psicoterapia analítica. En Obras completas (vol. XVII). Amorrortu.

FREUd, S. (1988e). Índices y bibliografías. En Obras completas (vol. xxIV). Amorrortu.

Grinberg, L. y Grinberg, R. (1976). Identidad y cambio. Paidós.

LANGer, M. (1984). Memoria, historia y diálogo psicoanalítico. Folios.

Laplanche, J. y Pontalis, J. B. (1971). Diccionario de psicoanálisis. Labor.

SchKolnik, F. (1987). Abstinencia y transgresión. Revista uruguaya de psicoanálisis, 65. www.apuruguay.org/apurevista/1980/16887247198765 02.pdf

* Las referencias bibliográficas y las citas están tomadas de la publicación original y no se han ajustado a las normas de referenciación bibliográfica vigentes. 


\section{ADENDA (JULIO DE 2021)}

La temática de este artículo está muy vigente en la actualidad por dos razones. Por un lado, hace a la actividad clínica de todos los días y, por otro, la formulación freudiana de estos dos conceptos ha devenido en objeto de innumerables discusiones y miradas críticas en los últimos tiempos.

Jorge Rosa centra su análisis en la incidencia de la ideología del terapeuta, en su concepción del psicoanálisis y en su identidad como terapeuta. Y considera también tanto el valor de las intervenciones, como los criterios que maneja acerca de la cura.

La abstinencia es una posibilidad técnica, una cierta posibilidad, que siempre es imprescindible considerar. La neutralidad es una ficción que entraña un peligro, ya que desconocerla como ficción nos puede llevar a ser «menos neutrales».

Ya hace mucho tiempo que en las bibliografías no figuran autores que acá son tomados como referentes, como Robert Castel, León y Rebeca Grinberg y también —aunque menos ausentes- Willy y Madeleine Baranger, sobre todo por ese gran aporte que fue Problemas del campo psicoanalítico, de 1969.

Seguramente, en este artículo el autor está optando por una conceptualización particular de estos términos, ligada a las ideas predominantes en determinado tiempo histórico, de las que es difícil excluirse.

En las últimas décadas del siglo xx hemos asistido a un cambio de paradigma en la concepción del sujeto psíquico. La diferencia sexual, centro de estudios e investigaciones del psicoanálisis, tanto en la teoría como en la clínica durante el último siglo, quedó ubicada como uno de los componentes que dan acceso a la categoría de sujeto. Este cambio epistemológico ubicó a la intersubjetividad como paradigma del origen y la estructuración del sujeto psíquico y llevó a revisitar tanto el concepto 
de abstinencia como el de neutralidad, así como el encuadre y el lugar de las intervenciones del terapeuta.

Numerosos autores han investigado acerca de la influencia mutua de la díada analítica y han aportado varias líneas de pensamiento, que van desde la teoría del apego y los procesos vinculares, hasta las neurociencias. Este artículo, escrito a los nueve años de la fundación de AUDEPP, nos deja la pregunta de cuál sería el pensamiento del autor hoy, pasados cuarenta años.

Pensamos que hubiera enfrentado el desafío de los tiempos actuales, asumiendo los cambios de paradigma en la ciencia y en el psicoanálisis, y que hubiera apuntado a establecer la flexibilidad necesaria para asumir estos cambios en el trabajo analítico.

También pensamos que, felizmente, ese es el camino por el que transita hoy la institución. 